\title{
LA AVIFAUNA DE LOS EMBALSES DE «EL HONDO» (ALICANTE)
}

Por José-Damián Navarro Medina y José Navarro García.

\section{INTRODUCCIÓN.}

En el marco de la problemática actual de la conservación de la Naturaleza, destaca por su gran importancia la preservación de las zonas húmedas naturales, uno de los biotopos más ricos en nuestro planeta. A tal fin, se han realizado numerosos acuerdos de ámbito internacional, al objeto de homogeneizar la acción protectora, entre los que destacan el Proyecto MAR (1962), la Conferencia Internacional sobre Conservación de Zonas Húmedas y Aves Acuáticas de Ramsar (1971) y la Conferencia Europea de Protección de Aves Acuáticas de Noorwijk aan Zee (1966). En el citado Proyecto MAR se incluyen a los Embalses de «El Hondo» como prioritarios de conservación.

En los países circunmediterráneos y sobre todo en España, las zonas palustres naturales siempre han sido consideradas lugares insanos a los que había que rescatar para la agricultura lo que, unido a la necesidad de erradicar el paludismo y bajo el auspicio de la aún no derogada Ley Cambó, ha supuesto la desaparición durante el presente siglo de las lagunas de La Janda, Antela, La Nava, El Calderón y gran parte de las Tablas de Daimiel. Aún hoy, cuando las teorías proteccionistas y ecologistas empiezan a generalizarse entre la opinión pública, la totalidad de nuestras zonas húmedas, sea cual sea su tamaño e importancia ecológica, están amenazadas por la contaminación, las autopistas, las urbanizaciones, la caza abusiva, etc., etc. 
Los embalses de «El Hondo» presentan una importancia zoológica y ecológica indudable. Durante nueve años, venimos estudiando su rica avifauna acuática y palustre, habiendo comprobado la irrenunciable necesidad de que dicho paraje permanezca.

\section{ASPECTOS GENERALES DEL MARCO ECOLÓGICO.}

Desde Albatera y la Sierra de Callosa hasta el Mediterráneo en Santa Pola, se halla la zona denominada Saladares de Alicante, cuyo suelo hidromorfo con muchos limos es muy salino e impermeable. La mayoría de la zona, debido a desecaciones llevadas a cabo durante siglos (autor destacado fue el Cardenal Belluga), se encuentra en la actualidad bajo aprovechamiento agrícola.

Entre Elche, Catral y Dolores, desde siempre, unos cuantos miles de hectáreas se encharcaban todos los ínviernos, tanto con aguas provenientes de la cercana Sierra de Crevillente como por afloramientos de la capa freática. Durante el verano, sin embargo, el paraje permanecía casi totalmente seco, cubierto de Salicornias y otras plantas halófilas. Estos encharcamientos invernales de considerable extensión, propiciaron la aparición de inmensas cantidades de aves acuáticas, que hicieron de «El Hondo» un conocido cazadero de anátidas ya en la Edad Media (don Juan Manuel lo cita en su Libro de la Caza).

A mediados del presente siglo, se construyeron en dicho marjal dos embalses, al objeto de utilizarlos para el riego a base de aguas del Segura, lo que se viene haciendo hasta la actualidad.

Los Embalses de «El Hondo», que pertenecen a la Comunidad de Riegos de Levante, ocupan una extensión de unas 1.650 Has., incluyendo en la misma los embalses de Poniente y Levante (1.100 Has. entre ambos), dos Charcas naturales y el terreno palustre circundante. Se encuentran a unos $8 \mathrm{Kms}$., al sur de Elche y a unos 4 al este de Crevillente. (Fig. 1).

El terreno constituye una depresión natural con cotas de 2 a 5 metros sobre el nivel del mar. Los embalses se sitúan a ambos lados del Canal Principal o de Levante, que los une y separa al mismo tiempo, con el que se comunican mediante cinco grupos de compuertas. Las dos charcas anexas a los embalses, de aguas salinas procedentes de manan- 


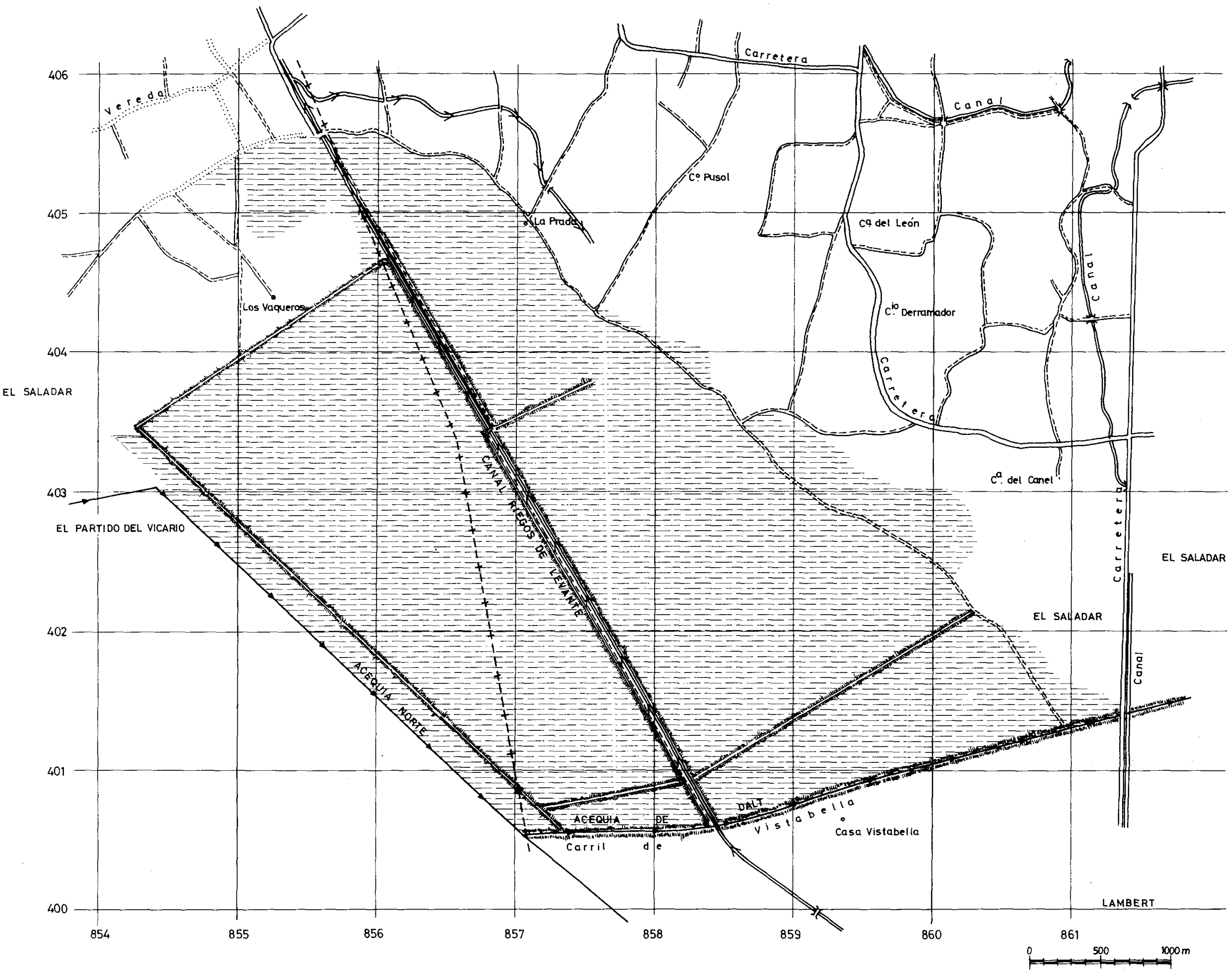


tiales naturales, no participan del régimen de riegos, por lo que las fluctuaciones de nivel de sus aguas son menos extremas, presentando éstas una mayor transparencia y pureza.

Al sur de «El Hondo», a unos $20 \mathrm{Kms}$., en línea recta, se encuentran las Salinas de la Mata y Torrevieja, y a $11 \mathrm{Kms}$., al este, las de Santa Pola y Albufera de Elche, formando así un conjunto palustre de indudable valor ecológico y faunístico.

Tanto las aguas de los embalses como la de las charcas, llevan en disolución cloruros en distinta concentración; mientras que las primeras presentan concentraciones salinas entre 0,4 y 1,0 grs., de $\mathrm{ClNa}$. por litro, las segundas alcanzan 7,0 y 9,0 g. $\mathrm{CINa} / 1$.

El agua embalsada, al proceder de zonas de riego tratadas con fertilizantes, tiende a la eutrofización, causa por lo que el carrizal aumenta en extensión rápidamente, debiendo ser cortado con periodicidad. Sin duda, dichas aguas también presentarán concentraciones de todo tipo de plaguicidas, lo que sin duda debe ser la causa de una apreciable, aunque lenta, desaparición de la ova que cubre, en verano, grandes superficies de agua.

Aparte de la explotación agrícola, «El Hondo» también es aprovechado por su caza y pesca, actividades éstas que, siendo cuidadosamente planificadas y controladas, pueden ser compatibles con la conservación del ecosistema.

La especie botánica dominante es, indudablemente, el Carrizo (Phragmithes communis), que se convierte en el perfecto cobijo de la inmensa mayoría de aves. Otras especies, escasamente representadas, son Arundo dDonax, Thypha angustifolia, Cyperus glaucus, Tamarix gallica, Salicornia spp. y Salsosa spp., etc. En el camino que, junto con el Canal Principal separa a los embalses, encontramos Eucaliptus spp., Phoenix spp., etc. En cuanto a algas, están presentes varias especies de Cianofíceas, Dinoflageladas, Diatomeas y Euclorofíceas; la C.R.L. cita, además, Rhizoclonium rivulare (recesivo) y Chara officinalis.

Atendiendo a la vegetación, podemos clasificar «El Hondo» en cuatro hábitats diferentes: aguas abiertas, carrizal, marjal y saladar. 
Aparte de las especies de aves observadas, motivo de este trabajo, y antes de entrar de lleno con ellas, queremos reseñar otras especies de cordados detectados por nosotros en «El Hondo»:

PECES: Cyprinus carpio, A. anguilla, Mugil spp., Gambusia affinis, Atherina mochon, C. carassius, Barbus spp. y Afhanius iberus.

ANFIBIOS: Rana ridibunda, Bufo spp.

REPTILES: Malpolon nompessulanus, N. natrix, Natrix maura, Psammodromus algirus, Lacerta lepida, Acanthodactylus erythryrus.

MANÍFEROS: Arvicola sapidus, $R$. rattus, Rattus norvegicus, Apodemus sylvaticus, Mustela nivalis, Crocidura russula, Talpa spp., Erinaceus europaeus, $P$. pipistrellus, V. vulpes.

\section{MÉTODOS DE ESTUDIO.}

El método de estudio principal se basa en la observación directa, por medio de ópticas apropiadas: prismáticos 12 x 50 y catalejo de hasta $60 x$. La observación directa ha permitido catalogar prácticamente a la totalidad de especies que mencionamos después, exceptuando unas cuantas que hemos recogido de la bibliografía disponible y las efectuadas por otras personas, inéditas.

Se puede decir que se ha recorrido, durante el tiempo que venimos estudiando los embalses, la práctica totalidad del terreno que forma el coto, a excepción, lógicamente, de aquéllas zonas de impenetrable carrizal.

Una dififultad grave que hemos comprobado, sobre todo al intentar hacer taxiados de poblaciones nidificantes, es que al ser el $90 \%$ del paraje masa acuosa, y la mayoría de los nidos se encuentran sobre ella o en el carrizal, se nos hace sumamente difícil su hallazgo y observación. El recuento de nidos o parejas sólo es posible, a priori, para aquéllas aves que los construyen colonialmente y al descubierto, como Fumareles, los cuales los sitúan sobre el manto de algas flotantes y en sitios despejados.

En las observaciones, así como en los censos de aves acuáticas y 
campañas de anillamiento, han colaborado con nosotros diversos colegas con más o menos asiduidad y que, al ser demasiados, omitimos detallar no sin reiterarles desde estas líneas nuestro agradecimiento por su ayuda.

En los censos de aves acuáticas invernantes, de los que hasta la fecha sólo hemos realizado siete (ver más adelante), hemos puesto en práctica el sistema de recorrer, en sendas embarcaciones, ambos embalses al mismo tiempo, para evitar en lo posible repeticiones que, en principio, no han tenido lugar o, como máximo, han sido de poca importancia, puesto que se muestra claramente una preferencia zonal distinta entre patos nadadores y buceadores. Así, los primeros se concentran principalmente en la zona norte del embalse de Poniente, por ser en la que existe menor profundidad de agua, lo que aprovechan para zambullirse y recoger las plantas del fondo sin dificultad. En cambio, los patos buceadores prefieren el embalse de Levante, de mayor profundidad, y en el que se sitúan en el centro, para divisar perfectamente cualquier peligro, real o ficticio, y tener suficiente espacio para su lento y trabajoso despegue.

Respecto a los anillamientos, se han seguido dos sistemas diferentes: primeramente, la colocación de redes japonesas, normalmente dos y en ocasiones hasta cinco, en diversos puntos del carrizal, quedando atrapados variedad de paseriformes.

El segundo sistema consiste en el anillamiento de pollos en época de cría, principalmente de Fumareles, a los que se les coge flotando cerca del nido o, a veces, incluso lejos de él. La tarea es difícil, puesto que debido a la gran cantidad de ova existente en esa época, la navegación se hace sumamente dificultosa, a pesar de lo cual se consiguen muy buenos resultados gracias a la experiencia de los barqueros en el manejo de la pértiga. Algunos años, sin embargo, al eclosionar los huevos después de fin de junio, había muy poco nivel de agua y mucha ova, por lo que los resultados fueron mínimos. Además de los pollos de Fumarel, que pululan en abundancia, también se consiguen atrapar, en reducido número, de otras especies de aves acuáticas.

El estudio de los embalses lo comenzamos en otoño de $1968 \mathrm{y}$, hasta la fecha, hemos realizado más de 190 visitas de estudio, que si las consideramos a una media de cinco horas por cada una, nos dan un total de casi 1.000 horas de observación. 


\section{RESULTADOS DE LOS ANILLAMIENTOS.}

A continuación, se da una lista por especies, de las aves anilladas en «El Hondo» durante nuestro estudio, y hasta la fecha. Todos los anillamientos se han realizado de 1972 a 1977 . La mayoría de ellos han sido efectuados por el Equipo de Anillamiento del Sureste (EQASE), dependiente del Centro de Migración de la Sociedad Española de Ornitología.

\section{ESPECIE}

Podiceps ruficollis................................... 3

Podiceps cristatus.................................... 2

Ixobrychus minutus..................................... 3

Egretta garzetta...................................... 93

Ardeola ralloides........................................ 21

Ardea purpurea........................................... 4

Aythya ferina............................................ 2

Netta rufina............................................. 2

Gallinula chloropus...................................... 2

Fulica atra.............................................. 8

Himantopus himantopus............................... 13

Chlidonias hybrida....................................... 1.107

Alcedo atthis............................................ 1

Hirundo rustica............................................. 16

Motacilla flava......................................... 2

Cettia cetti............................................. 5

Acrocephalus arundinaceus............................. 29

Acrocephalus schoenobaenus............................ 1

Acrocephalus melanopogon.............................. 2

Acrocephalus scirpaceus................................ 92

Sylvia atricapilla....................................... 4

Sylvia communis......................................... 2

Phylloscopus collybita................................... 24

Saxicola torquata...................................... 3

Erithacus rubecula......................................... 7

Panurus biarmicus....................................... 3

Parus major............................................. 1

Luscinia megarhynchos................................. 1 
Remiz pendulinus........................................ 1

Serinus serinus.......................................... 1

Carduelis carduelis.......................................... 5

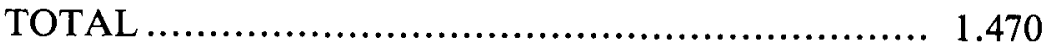

TOTAL ESPECIES: 32

RESULTADOS DE LOS CENSOS

\begin{tabular}{|c|c|c|c|c|c|c|c|}
\hline \multirow[b]{2}{*}{ ESPECIES } & \multirow[b]{2}{*}{$\mathrm{I}-72 *$} & \multicolumn{2}{|c|}{ MES Y AÑO } & \multicolumn{4}{|c|}{ CANTIDADES-DÍA } \\
\hline & & I-73 & $\mathrm{X}-73$ & XI-73 & $\mathrm{I}-75$ & I-76 & III-76 \\
\hline Podiceps ruficollis........................ & 40 & 95 & 150 & 60 & 13 & 15 & 1 \\
\hline Podiceps nigricollis.................... & & 23 & & & & & \\
\hline Podiceps cristatus..................... & & & 2 & & & 14 & 20 \\
\hline Phoenicopterus ruber................. & & & 47 & 80 & 2 & & \\
\hline 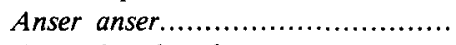 & & & & 1 & 18 & & \\
\hline Anas platyrhynchos..................... & 4 & 15 & 310 & 30 & 2 & 20 & 20 \\
\hline Anas acuta............ & & 570 & 850 & 300 & 280 & 114 & 45 \\
\hline Anas penelope..... & & 300 & 1.200 & 1.800 & 850 & 267 & 2 \\
\hline 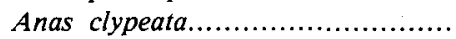 & 350 & 950 & 440 & 1.470 & 745 & 1.400 & 190 \\
\hline 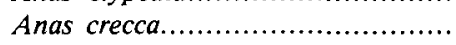 & & & 200 & 2 & 2 & 115 & 3 \\
\hline 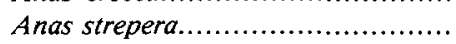 & 25 & 10 & 20 & 200 & & 12 & 21 \\
\hline 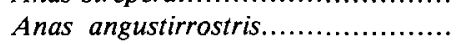 & & & & 1 & & & \\
\hline Anas querquedula..................... & & & & & & & 35 \\
\hline 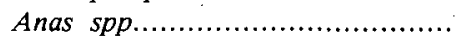 & & & & & & 200 & \\
\hline 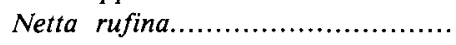 & 1.500 & 1.150 & 2.060 & 2.000 & & 750 & 216 \\
\hline Aythya ferina............................ & 15 & 3.560 & 8.200 & 3.850 & 1.150 & 6.120 & 556 \\
\hline 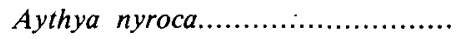 & & 2 & & & & & \\
\hline Aythya fuligula...................... & & & 1 & & 7 & 3 & \\
\hline 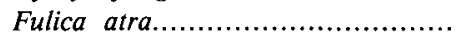 & 800 & 3.900 & 12.100 & 8.300 & 4.700 & 5.980 & 1.010 \\
\hline Phalacrocorax carbo................... & & & & & 1 & & \\
\hline TOTALES & 2.734 & 10.575 & 25.580 & 18.114 & 7.770 & 15.010 & 2.136 \\
\hline
\end{tabular}

* Censo parcial, referido sólo al Embalse de Poniente. 


\section{LAS AVES}

Más adelante, ordenada sistemáticamente, se da la lista completa de las especies de aves acuáticas y palustres (excepto algunas que no lo son, pero que se indican de forma desarrollada por su interés) observadas en «El Hondo», ya por nosotros mismos o por citas bibliográficas. El total de especies del primer apartado es de 80 . Al final, se incluye la lista del resto de especies vistas, que comprende 53 más. En este primer trabajo se incluyen aproximadamente la mitad de las especies. En fecha reciente y en la misma revista aparecerá el resto.

Sin embargo, previamente a lo citado, damos a continuación una clasificación de las especies más representativas, divididas en tres grandes grupos: sedentarias, indígenas estivales e invernantes. Están ordenadas de acuerdo al índice de Rendimiento Específico (que es el porcentaje de veces vista sobre el número de visitas consideradas), que indica, más que su abundancia real, su facilidad de observación, aunque en general ambos conceptos están íntimamente relacionados (por ejemplo, las especies que son más escondedizas, dan un índice de Rendimiento mucho más bajo que su abundancia real — Rascón, Zampullín Cuellinegro, Porrón Pardo, Carricerín Real...-).

SEDENTARIAS (consideradas 75 fechas en toda época).

\section{ESPECIE}

R.E.

$\%$

Focha Común.

97,30

Polla de Agua.

94,70

Tarabilla Común.

88,00

Zampullinn Chico.

86,70

Pato Colorado

80,00

Porrón Común.

80,00

Golondrina Común....................................... $\quad \mathbf{7 4 , 7 0}$

Buitrón................................................. $\quad 69,30$

Gorrión Común.......................................... $\quad 66,70$

Jilguero ................................................... $\quad 64,00$

Garceta Común......................................... $\quad 52,00$

Somormujo Lavanco..................................... $\quad 49,30$ 
Bigotudo............................................... $\quad 44,00$

Ánade Real.............................................. $\quad 37,30$

Carbonero Común........................................ $\quad 34,70$

Ánade Friso.............................................. $\quad 26,70$

Cerceta Pardilla............................................ $\quad 26,70$

Pájaro Moscón........................................... 22,70

Escribano Palustre......................................... 20,00

Rascón................................................... $\quad 13,30$

Carricerín Real......................................... $\quad 10,70$

Parrón Pardo............................................. $\quad 5,30$

INDÍGENAS ESTIVALES (consideradas 23 fechas, de Abril a Julio).

Carricero Común........................................... $\quad 91,30$

Carricero Tordal........................................ $\quad 87,00$

Fumarel Cariblanco....................................... $\quad 87,00$

Cigüeñuela ........................................... $\quad 82,60$

Avetorillo Común.......................................... $\quad 82,60$

Garza Imperial........................................... $\quad 69,60$

Zampullín Cuellinegro................................... $\quad 47,80$

Charrancito .............................................. $\quad 43,50$

Lavandera Boyera....................................... $\quad 34,80$

Canastera................................................. $\quad 34,80$

Garcilla Cangrejera....................................... $\quad 30,40$

Fumarel Común........................................... 21,70

Buscarla Unicolor....................................... $\quad 17,40$

Chorlitejo Patinegro....................................... $\quad 13,00$

INVERNALES REGULARES (consideradas 36 fechas, de Octubre a Febrero).

Estornino Negro........................................ $\quad 83,30$

Ruiseñor Bastardo......................................... $\quad 80,60$

Mosquitero Común......................................... $\quad 75,00$

Lavandera Blanca...................................... $\quad 75,00$

Petirrojo.................................................. $\quad 72,20$

Aguilucho Lagunero........................................ $\quad 69,40$

Gaviota Reidora.......................................... $\quad \mathbf{6 6 , 7 0}$

Pato Cuchara.............................................. $\quad 61,10$

Pinzón Vulgar.......................................... $\quad 52,80$ 
Agachadiza Común...................................... $\quad 50,00$

Bisbita Común........................................... $\quad 41,70$

Ánade Silbón.............................................. $\quad 38,90$

Avión Roquero......................................... $\quad 38,90$

Lavandera Cascadeña...................................... $\quad 36,10$

Avefría...................................................... $\quad 36,10$

Ánade Rabudo.......................................... $\quad 36,10$

Bisbita Ribereño.......................................... $\quad 33,30$

Garza Real............................................ $\quad 33,30$

Flamenco................................................ $\quad 19,40$

Alcaudón Real............................................. $\quad 16,70$

Cormorán Grande........................................ $\quad 2,80$

\section{I) ESPECIES ACUÁTICAS Y PALUSTRES.}

Se relacionan sistemáticamente y de manera desarrollada, indicando tanto su status como todo lo que creemos interesante sobre cada una de las especies. En la cabecera, se cita nombre científico, nombre técnico en español (según lista patrón de la Sociedad Española de Ornitología) y nombre o nombres vernáculos (cuando lo tiene y se conoce). Las especies precedidas del signo $x$, indican nidificación comprobada satisfactoriamente; las precedidas del signo :, las de nidificación posible y teóricamente indudable. A continuación del nombre vernáculo, se usarán los siguientes símbolos:

\section{$S$, sedentario $\quad E$, estival $\quad I$, invernante $m P$, migrante primaveral $m O$, id. otoñal $D$, divagante a partir de biotopos cercanos}

X Podiceps ruficollis, Zampullin Chico.-(Sapo, Sapet)

Especie indígena y nidificante, común pero sin abundar. Anida principalmente en el embalse de Poniente, como ya señaló MARTORELL (1966); también, en menos cantidad, en las Charcas. Construye pequeño nido flotante a base de plantas en descomposición, cerca de las orillas y anclado entre carrizos, siempre en aguas de poca profundidad. Nidos normalmente con tres-cuatro huevos, blancos al principio y que van tornándose amarillentos conforme progresa la incubación. Hemos observado en algunas ocasiones, el hábito de esta especie de tapar el nido, al abandonarlo por sentirse alarmado, con algas en descomposi- 
ción por medio de un rápido movimiento de patas, zambulléndose inmediatamente, y haciendo prácticamente invisible

Se ven ya con plumaje nupcial a principios de marzo. Nidos con puesta a finales del mismo mes. Parejas acompañadas de pequeños pollos - dos o tres-, en abril, mayo y junio.

En invierno permanece la misma población nidificante, recibiéndose en poca cantidad invernantes foráneos, como dijimos ya anteriormente (NAVARRO, 1972). Durante esa época, se vuelve más confiado, viéndosele en aguas descubiertas, al contrario que durante la época de cría, en que se encuentra siempre en el carrizal o no muy lejos de él.

Un spm. anillado el 13.08.72, da las siguientes medidas (en mm.): ala, 71; tarso, 32; cúlmen, 15.

En dos ocasiones lo hemos visto, excepcionalmente, en vuelo, cosa que normalmente nunca sucede, ya que prefiere sumergirse ante cualquier peligro.

Según escasas observaciones al respecto, la alimentación debe ser básicamente, invertebrados acuáticos.

X Podiceps nigricollis, Zampullín Cuellinegro.-(Cabre)

Especie mucho más escasa que la anterior en todo tiempo. Status principal estival, aunque la mayoría de los nidificantes permanecen durante el invierno, e incluso a veces se observa una clara afluencia de visitantes extra-hondeños. En otras zonas cercanas, sin embargo, hemos podido comprobar su invernada masiva, quizá como dato inédito para Iberia (NAVARRO y otros, en ARAUJO \& GARCÍA RUA, 1973); nos referimos a las Salinas de La Mata, en las cuales contamos un mínimo de 3.500 spms. el 11.01.73.

En junio de 1965, no es observado por MARTORELL (1966), aunque sí indica que es bien conocido por los pescadores del lugar.

Se ven normalmente de marzo a septiembre, siempre en escasa cantidad, y generalmente en parejas, con su bello plumaje nupcial. Mucho más confiado que $P$. ruficollis, ya que no se zambulle ante el observador, dejándole acercarse mucho. 
Nidifica en nidos construidos sobre vegetación flotante, ya sea entre las colonias de Fumarel o muy cerca de ellas (lugares con más profundidad de agua y con menos cobertura que $P$. ruficollis). Nidos normalmente con cuatro huevos; al parecer, no tapan éstos al abandonarlos, en contra de la especie anterior, pero la escasez de observaciones al respecto no nos permiten afirmarlo. Vemos pollos principalmente en mayo, algunos de ellos transportados sobre el dorso de sus padres. El 5-5-74, vemos una pareja seguida de cuatro pequeños; los padres se zambullen y emergen constantemente, cada vez trayendo en el pico un pequeño pez ( $₫$ Atherina?), que dan a sus hijos; posiblemente, la alimentación de la especie incluya un mayor porcentaje de peces que la de su afín.

Al parecer, en franca regresión en estos últimos tres años.

X Podiceps cristatus, Somormujo Lavanco.-(Gallo, Gall)

Especie sedentaria y relativamente escasa. Quizá aniden unas 15 parejas en todo el coto. Mayor contingente en época estival, pues en invierno se ven muy pocos; quizá la mayoría inicien una dispersión postgenerativa una vez acabada la nidificación.

Hemos visto nidos con huevos a primeros de junio (en una ocasión hasta cinco en muy poco espacio). Muchas parejas con pollos, éstos junto o sobre los adultos, de mayo a julio-agosto.

Las bellas y espectaculares paradas nupciales empiezan muy pronto, ya a finales de febrero; la mayoría de los spms. observados en época de cría ha sido cerca del camino y en las Charcas, por lo que suponemos que, al igual que $P$. ruficollis, prefieren aguas someras para la ubicación del nido. En raras ocasiones, individuos en vuelo.

Últimamente, con la instalación de redes semi-espirales para la captura de carpas, hemos observado una querencia cada vez mayor de esta especie por pescar junto o dentro de dichas trampas.

Phalacrocorax Carbo, Cormorán Grande.-(Corveta)

Visitante invernal esporádico, que se presenta aquí de vez en cuando quizá procedente de las cercanas costas. La mayoría de las ocasio- 
nes lo hemos visto en la Charca Sur. Acostumbra a posarse en las estaquillas de sujección de las redes, al acecho de los peces que pasan. $\mathrm{Su}$ cantidad, de una vez a otra, varía de dos a ocho spms.

Phalacrocorax aristotelis, Cormorán Moñudo.-(Corveta)

El guarda D. Manuel Berenguer nos informó que 3 spms., de esta especie fueron vistos durante más de una semana, a finales de noviembre de 1972, en el embalse de Poniente, posados en estaquillas.

Botaurus stellaris, Avetoro Común

No tenemos citas personales, ni visuales ni acústicas, a lo largo de las 190 visitas efectuadas, por lo que dudamos que en la actualidad forme parte de la avifauna hondeña. Lo referimos por la cita indirecta que recoge MARTORELL (1966): «Según el Sr. Navarro se encuentra en la laguna y nidifica. No pudimos comprobar su presencia». Ésto data de junio de 1965. Lo extraño es que, en contra de nuestras nulas observaciones, el hábitat y la zona son por completo propicias a la nidificación o mera presencia de esta especie.

X Ixobrychus minutus, Avetorillo Común.-(Punchabous)

E

Especie típicamente estival y nidificante francamente común. Llegan los primeros a principios de abril, y se ven hasta mediados de septiembre.

Aparentemente, hay mayor proporción de machos que de hembras. Ya fue citado por MARTORELL (1966) como abundante.

Anida en lo más intrincado del carrizal. Nosotros pudimos observar un nido y anillar sus pollos el 29.06.73 (hasta ahora la única prueba directa de nidificación). Dicho nido era muy pequeño, construido entre carrizos, directamente sobre la superficie del agua; lados bajos y muy abierto. El 21.06.73 contenía 2 pulls., recién nacidos (plumón suave color salmón) y tres pequeños huevos blancos, muy redondeados. El 29, lo visitamos de nuevo y tenía dos pulls, muy grandes, que después de anillados escaparon trepando por los carrizos, otros dos en plumón uno de ellos muy pequeño para ser anillado-y un nuevo sin eclosionar. 
Aparte de ésto, todos los años vemos adultos que salen a toda prisa de determinados puntos del carrizal o que se aquerencian a ellos, demostración típica de poseer nidos.

Es frecuente observar a ejemplares posados en tranquilos remansos, y en las compuertas y lados del canal, al acecho de sus presas.

Principalmente durante ambos pasos, como migrante sedimentado que permanece en «El Hondo» los días precisos para reponer energías. MARTORELL (1966) lo cita en junio-65: «vistos varios spms. volando entre un bando de garzas. No obstante, no pudimos encontrar nido en la colonia de ardéidas». Exactamente lo mismo que a Martorell nos pasó a nosotros este año, ya que de la colonia de garzas que descubrimos en el mes de junio, vinos salir hasta 6-8 spms. volando, y a pesar de ello no encontramos ningún nido de Martinete.

Las fechas de observaciones de migrantes sedimentados, los cuales aparecen todos los años, son la $3^{a}$ decena de marzo, para el paso primaveral, y la primera quincena de septiembre para el otoñal, aunque algunos años se retrasa algún ejemplar (p. ej.: 1.11.72).

Normalmente se ven en pequeños bandos de 3 a 7 spms., siempre muy aquerenciados a los eucaliptos - principalmente a los de la parte sur - en los cuales se posan a descansar. El mayor número lo observamos el 27.03.74, con una cantidad de más de 30 ejemplares, todos adultos.

Indígena escasa, que no fue observada por Martorell, de la cual hemos podido comprobar por primera vez su nidificación en el presente año. Dentro de la colonia de Egretta que descubrimos en junio, en el embalse de Levante, había perfectamente estructurada, en el mismo centro de aquélla, una subcolonia de esta especie, compuesta por unos 20 nidos, muy cerca unos de otros (menos de un metro en ocasiones). Perfectamente sincronizados, todos estaban llenos de pequeños pulls., de edades muy similares. Los adultos entraban a cebar constantemente, a pesar de nuestra cercana presencia. Anillamos unos 22 pulls., pues 
muchos nidos estaban inaccesibles desde la barca, en medio de una espesa mata de carrizal.

Fechas de llegada a finales de abril o primeros de mayo. Partidas en septiembre, aunque estuvimos observando un ejemplar joven, en 1972, hasta mediados de noviembre - quizá un migrante despistado-.

Siempre se las ve en solitario o en parejas, posadas en las orillas del agua, al acecho de presas; algunas veces en zonas despejadas de carrizal, sobre fondo de tierra, lo que las hace totalmente invisibles desde lejos (NAVARRO, 1972).

Nidificación comprobada por MARTORELL (1966) en junio-65, citando dos nidos con pulls., recién nacidos y otros tres con huevos, además de bastantes adultos; pero, en la actualidad, no creemos que nidifique aquí - no la hemos visto nunca en época de cría- y sólo la hemos observado en contadas ocasiones en época invernal (errantes). Por supuesto, no la vimos en la colonia de ardéidas descubierta este año.

Nuestras observaciones son las siguientes: 25.11 .73 , un bando de 23 ejemplares en plumaje invernal, en la orilla de los embalses y sobre el camino, 8.12.73, un ejemplar junto al camino, 11.12.73, un bando de 12 aves en vuelo. Algunas observaciones más, de pocos individuos, en inviernos posteriores.

\section{Egretta garzetta, Garceta Común.-(Garsotet)}

Nidificación ya citada por MARTORELL (1966) en junio-65, y que hemos podido comprobar nosotros este verano, aunque seguramente no crían aquí todos los años. Dice el autor aludido: «amplia profusión de nidos y pollos en el espeso carrizal, de todas las edades. Se anillan 41 ». La colonia se ubicaba entonces en el carrizal Este del embalse de Levante; no da datos cuantitativos de su envergadura.

En junio de este año la colonia se encontraba en el carrizal Oeste del embalse de Levante, y en un $90 \%$ la componía esta especie (el $10 \%$ restante, $A$. ralloides y $A$. purpurea). Decenas de pollos de crecido tamaño trepaban por los carrizos ante nuestra presencia. Algunos nidos 
con huevos, y otros con pollos aún pequeños. En menos de dos horas pudimos atrapar y anillar más de 90 . Estimamos, por encima -debido a que quizá muchos nidos sé nos hacían invisibles-, un mínimo de 100 parejas nidificantes.

Aparte de esto, Egretta es de presencia regular y fluctuante en cantidad durante todo el año. En invierno quizá se vea incrementada con invernantes la población indígena, que además tiende a la dispersión postnupcial. Acostumbran a posarse sobre las salicornias de la Charca Norte. Abundante como invernante en las Salinas de Santa Pola (en invierno parece ser que prefieren zonas despejadas) (NAVARRO, 1971b).

Ardea cinerea, Garza Real.-(Garsa)

$\mathrm{mO}(\mathrm{I})$

Típico invernante que llega en septiembre (considerables concentraciones de migrantes) (NAVARRO, 1972) y que marcha en abril, aunque siguen viéndose raros ejemplares hasta final de mayo (idem).

Siempre se las ve en vuelo sobre los carrizales y echándose entre ellos. También les gusta posarse en la Charca Norte, al igual que la especie anterior. Mayores cantidades en las dos siguientes fechas: 26.09.71, unos 10 spms. juntos, 28.10.73, unos 40 ejemplares en vuelo arremolinado, Charca Sur-Este.

En otras zonas húmedas cercanas existen grandes concentraciones invernales. Por ejemplo, en las salinas de Santa Pola no son raras cantidades de 50-70 aves en corto espacio, lo que puede suponer un total de casi 200 en todas las salinas.

X Ardea purpurea, Garza Imperial.-(Garsa)

$\mathrm{E}$

Indígena estival típico, que anida en moderada cantidad. MARTORELL (1966) encontró un nido en junio-65, entre la colonia de Egretta. Nosotros vemos, casi todas las primaveras, parejas que entran repetidas veces a determinados puntos del carrizal, con grandes tallos de carrizos secos en el pico. Además, todos los años, a final del verano, vemos jóvenes de la temporada.

En junio de este año, pudimos ver varios nidos — unos con huevos y otros con pollos - entre la colonia de Egretta que descubrimos en el 
embalse de Levante. Anillamos 4 pull. Suponemos que habría unos 10 nidos en la colonia.

Llega a partir de finales de marzo y se marcha a finales de septiembre. Observaciones generales a razón de 2-4 individuos/día, por lo que debe considerarse como especie escasa.

Platalea leucorodia, Espátula.$\mathrm{mO}$

El Sr. Miró, director de la Cía de Riegos de Levante, nos comunicó haber observado a dos individuos de esta bella especie en agosto de 1969, posados junto al canal. Sin duda, en dispersión postgenerativa.

Plegadis falcinellus, Morito.-

$\mathrm{mO}$

Según MARTORELL (1966): «el Sr. Navarro ha observado un individuo de esta especie este mismo verano», refiriéndose al de 1965. Igualmente, èn dispersión postgenerativa.

$X$ Phoenicopterus ruber, Flamenco.-(Flamenco)

MARTORELL vio un bando de 200 spms. durante tres visitas consecutivas en junio-65; según dicho autor, intentaron la nidificación, construyendo nidos, en la zona norte del embalse de Poniente, pero los bruscos cambios del nivel del agua malograron el intento.

Nosotros lo observamos a menudo, siempre en las Charcas Norte o Sur, salvo algunas raras ocasiones en que los hemos visto en el norte de Poniente, por ser zonas de poca profundidad. El 13.12.70, unos 150 spms. en la Charca Sur-Este (NAVARRO, 1972). Del orden de 40 a 80 spms. - variaban día a día-, durante el invierno 1973-74, en la Charca Norte. En estos bandos, iban de 4 a 12 juvs. del año, que se mantenían unidos entre sí y algo apartados del grueso de los adultos. Incluso pudimos observar, en dos ocasiones, iniciaciones de parada nupcial en varios adultos. En general, todos los otoños e inviernos en cantidades variables (éste háy unos 400 en Charca Sur).

De presencia regular en las Salinas de La Mata y Santa Pola, (Mac Ivor en NAVARRO 1972 y datos propios inéditos), donde anidan algunos años. 
Según los guardas del coto, de presencia más o menos regular todos los inviernos, aunqưe en reducido número. Ya en febrero de 1962, según D. Ginés Torres, se cazó un ejemplar de los tres que había ese año.

Nosotros lo hemos visto, siempre uno o dos ejemplares, en los meses de diciembre, enero y febrero de varios años, en la zona norte del embalse de Poniente y en la Charca Sur (los dos sitios con menos profundidad). Algunos años, los guardas nos informan haber observado hasta 30 ejemplares.

Anas platyrhynchos, Ánade Real.-(Collvert. Gabre -macho, Ánera -hembra-).

Especie sedentaria, que se ve incrementada suavemente por los invernantes $\mathrm{y}$, sobre todo, por los migrantes otoñales.

Anida en poca cantidad en los embalses, y sobre todo en las zonas de aguas someras del norte y del sur, entre salicornias según MARTORELL. En los meses de mayo y junio de casi todos los años, vemos hembras seguidas de polluelos (una de ellas con seis, NAVARRO 1972, el 31.05.70).

Una de las veces en que lo hemos visto en mayor cantidad, en octubre de 1973: más de 300 ejemplares, sin duda migrantes sedimentados; en invierno se caza en muy escaso número.

Anas crecca, Cerceta Común.-(Sarset, Sarseta).-

Invernante escasísimo, quizá debido a la demasiada profundidad de las aguas durante el invierno, siendo conocido que es un pato típico de aguas someras (CORONADO y otros, 1973), donde puede alcanzar el alimento con facilidad.

La única ocasión en que lo vimos en relativa cantidad, fue a finales de octubre de 1973: 200 spms. en la parte sur del embalse de Levante, sin duda migrantes sedimentados. 
Indígena, que nidifica en muy escasa cantidad y seguramente desde hace sólo unos cuantos años. Invernante en moderada cantidad, pero regular.

Parejas apareadas en mayo y junio (NAVARRO, 1972). Una hembra seguida de 9 pollos muy pequeños, a finales de junio de 1973, en el embalse de Poniente. Varias parejas en vuelo en varios años siguientes (meses de mayo y junio).

Observaciones invernales a razón de 2-20 individuos-día de media, excepto en 25.11.73, en que se contaron unos 200 (sin duda, de paso).

Preferencia marcada por el carrizal de aguas más o menos someras, de las orillas de los embalses y en las charcas.

Anas penelope, Ánade Silbón.-(Píulo, Silbón)

Invernante típico y regular, con poblaciones relativamente importantes, aunque algo fluctuantes.

Querencia a concentrarse principalmente en la zona norte del embalse de poniente (escasa profundidad). También en la Charca Sur y zona Este del embalse de Levante, aunque en mucha menor cantidad que en el primero.

Concentraciones importantes -máximos recuentos- en: 11.01.73, 300 spms.; 28.10.73, 1.200 spms.; 25.11.73, 1.800 spms.

Cazado en relativa cantidad, sobre todo en el embalse de Poniente. Observaciones invernales de noviembre a febrero (algunos años ya a finales de octubre). También lo hemos visto, en muy pequeña cantidad, en las cercanas Salinas de Santa Pola.

El 26.11.72, vimos recién cazado un macho de esta especie, que podría ser un híbrido con otro Anas spp. (posiblemente $A$. crecca), o de plumaje aberrante. Las diferencias que observamos con el típico macho de $A$. penelope fueron las siguientes: espejuelo verde sólo en los bordes exteriores de las secundarias $4^{\mathrm{a}}, 5^{\mathrm{a}}, 6^{\mathrm{a}}$ y $7^{\mathrm{a}}$, en vez de tenerlas 
todas de dicho color; el blanco de las supracobertoras alares era de un tono sucio y jaspeado de parduzco, que se difuminaba gradualmente con los otros colores del ala. Mancha verde muy conspicua en la cabeza, a modo de franja postocular, dividiendo los colores castaño y crema de aquélla.

Cuando se concentran, emiten en profusión sus silbidos característicos, que pueden ser oídos a mucha distancia.

Anas acuta, Ánade Rabudo.--(Rabejunc)

Invernante regular y normalmente escaso, que tiene las mismas marcadas preferencias zonales que la especie anterior.

Los primeros se ven, en escaso número a partir de mediados de octubre, y permanecen en los embalses hasta febrero-marzo todo lo más.

Las cantidades más importantes: $23.12 .72,120$ spms. en vuelo sobre la zona centro; 11.01.73, 550 spms. en el embalse de Poniente; 28.10.73, un total de 850 spms.; 25.11 .73 , unos $300 ; 25.1 .75$, unos 280 spms.; y 18.01.76, 114 spms. En todos los casos, prácticamente el $80 \%$ en el embalse de Poniente (zona norte).

La mayor cantidad, también en la zona citada, en 10.02.74, unos 1.000 spms. (migrantes sedimentados).

Al igual que $A$. penelope, se ven algunos en las Salinas de Santa Pola, pero siempre en cantidades inferiores a 40 spms./día.

Anas querquedula, Cerceta Carretona.-(Teulet)

$\mathrm{mP}$

Únicamente se presenta como migrante primaveral, y en reducido número. Casi todas las observaciones en el embalse de Poniente, cerca del camino, en aguas someras y muy cubiertas por el carrizal.

Observaciones de 2 a $10 \mathrm{spms}$./día, siempre en parejas (macho y hembra), en fechas comprendidas, con gran exactitud, entre 25 -febrero y 30-marzo de casi todos los años. Este otoño (octubre) pudimos ver, por primera vez, a un solo ejemplar en paso postnupcial. 
Uno de los invernantes más comunes y diseminados, siendo quizá el que mantiene, a grosso modo, las poblaciones menos fluctuantes, tanto mensual como anualmente.

Como en los otros ánades de superficie, prefiere la zona norte del embalse de Poniente y Charcas Sur, aunque también, por gustarle mayor cobertura palustre, se le ve - aunque en mucha menor cantidaden otras zonas de los embalses.

Primeras observaciones de invernantes a mediados de septiembre, permaneciendo hasta primeros de abril. Máximo de individuos de noviembre a enero, ambos inclusive.

Algunos años es la especie de pato más abundantemente cazada; por ejemplo, en el invierno de 1959-60 (TATO, 1960).

Máximos recuentos en las siguientes fechas: 21.01 .72 , más de 120 spms.; 23.01.72, 350 spms. en Poniente; 12.03.72, muy abundante, en bandadas enormes - quizá migrantes-; 11.01.73, 440 spms.; 25.11.73, 1.500 spms., principalmente en Poniente; 25.01.75, 750 spms.; 18.01.76, 1.400 spms.; y $21.03 .76,200$ spms.

También se le puede observar, en pequeños grupos de no más de 20 ejemplares, en la Charca Norte, bien nadando o posados en pequeñas elevaciones semi-secas, en medio del agua. Muy raro en las Salinas de Santa Pola.

Datos de anillamiento de un ejemplar capturado en «El Hondo»:
ANILLAMIENTO: 28-9-72, Bakerwooal (Holanda). CAPTURA: 02-2-75, «El Hondo» (Alicante). $\mathrm{N}^{\circ}$. ANILLA Y CENTRO: 5065978 - Arnehem.

Anas angustirostris, Cerceta Pardilla.-—(Rosseta)

Especie sedentaria, que nidifica en pequeña cantidad — datos indirectos- y que, debido a la fuerte característica vagante de esta especie, fluctúa considerablemente a lo largo del año, incluso de una semana a otra. 
No tenemos pruebas directas de nidificación, pero diversos guardas de la finca nos aseguran que la ven todos los años seguida de polluelos, muchos de los cuales caen al canal y son recogidos en la Elevación, devolviéndolos de nuevo al embalse. MARTORELL la cita, para junio-65, como «muy abundante; cría en gran profusión según el $\mathrm{Sr}$. Navarro».

Nosotros mismos, durante varias primaveras, hemos visto a diferentes parejas muy aquerenciadas a determinados puntos, durante varios domingos seguidos, lo que prácticamente las delata como propietarios de algún cercano nido.

Nuestras tres primeras citas de $A$. angustirostris en estos embalses, las señalamos ya en nuestro anterior artículo (NAVARRO 1972), pero como desde entonces hemos efectuado muchas más, nos abstenemos de relacionarlas todas. Observada también en zonas palustres cercanas, en épocas estival y primaveral (NAVARRO 1971b, 1971c y datos inéditos).

Como decíamos, su cantidad varía mucho a lo largo del año, y por no ser especie migratoria en nuestro país (CORONADO y otros, 1973) sólo es posible explicarlo por desplazamientos de aves nidificantes en otros lugares de la Península, que una vez finalizada la cría inician una dispersión más o menos errática. TRIGO DE YARTO (1960) la cazó en diciembre de 1959. PALAUS (1959) la cita como capturada abundantemente en noviembre de 1958. Nosotros la hemos observado, rara o escasa por lo general, en marzo, mayo, abril y junio, siempre en parejas más o menos aquerenciadas salvo excepciones (el 2.06.74, un bando de ca. 70 spms. en vuelo, Charca Sur-Este). Más abundante en septiembre, octubre, noviembre, diciembre y enero. En algunos días que hay cacería, algunos puestos cobran más Pardillas que cualquier otra especie de Anas (NAVARRO 1972).

La mayor abundancia de $A$. angustirostris la detectamos el 5.10.73, en que pudimos contar un mínimo de 400 , muy concentradas en unas aguas someras con salicornia, al norte de la Charca Norte. Por largo rato, en el crepúsculo, estuvieron entrando bandos de ellas, alguno de más de 50 aves. Posiblemente, procedentes de las Marismas del Guadalquivir donde, como todos sabemos, hubo una sequía atroz y gran mortalidad de aves por causas epizoóticas. 
En resumen, status general de sedentaria, con incremento de población principalmente a final de verano y otoño, $\mathrm{y}$ algo menos en el invierno, posiblemente a base de individuos erráticos o dispersivos a partir de otras zonas españolas.

$X$ Retta rufina, Pato Colorado.-(Sivert)

Especie sedentaria, que durante el invierno se ve incrementada con invernantes, posiblemente procedentes de zonas más o menos cercanas, y principalmente la manchega.

MARTORELL lo cita como «muy abundante» en junio de 1965. Nosotros lo vemos, al menos en moderada cantidad, en toda época (NAVARRO 1972), aumentando, como hemos dicho, en época invernal.

Ya se ven parejas formadas por ambos sexos a primeros de abril, e incluso antes. Hembras seguidas por pollos, de 5 a 12, durante todo el verano (y principalmente junio y julio).

Las mayores concentraciones en las siguientes fechas: 21.01.72, 200 spms.; 23.01.72, 1.500 spms.; 5.03.72, unos 500 spms. como mínimo en el embalse de Levante; $11.06 .72,100$ spms. en vuelo sobre Poniente; 8.10.72, aprox. 1.000 spms. en la Charca Norte; 12.11.72, idem.; $11.01 .73,1.250$ spms. en total; 21.06 .73 , unos 60 en la Charca Norte (¿mancones?); 5.10.73, 400 en la misma charca; 28.10.73, más de 2.000 en total; 25.11 .73 , unos 2.000 en total; 23.12 .73 , más de 1.000 en vuelo sobre la Charca Norte; 17.02.74, unos 600 en el centro del embalse de Poniente; 18.01.76, 750 spms. en total; 21.03.76, 216 spms.

En los días que hay cacería, la gran mayoría de los Netta hondeños huyen a refugiarse en el mismo centro de las Salinas de La Mata (Mac Ivor en NAVARRO 1972 y observaciones inéditas propias). Así, por ejemplo, el 16.01.72, se recontaron allí unos 3.200 spms. (Navarro y otros en BERNIS 1972 -donde por error de imprenta, se consignaron 320).

En la gran mayoría de ocasiones, esta especie se posa en zonas libres de carrizo, con mucha visibilidad y gran profundidad, para poder otear bien cualquier posible peligro; sin embargo, durante la época de nidificación, se esconde mayormente en el espeso carrizal. 
En general, misma consideración que para Netta rufina. Es decir, sedentario con pequeña población indígena, notablemente incrementada durante el invierno, aunque variando mucho de unas fechas a otras.

Estas fluctuaciones de invernantes, normalmente en espacios de tiempo relativamente cortos, quizá pueden ser explicadas por la movilidad invernal de esta especie; según datos recogidos en el Censo de Aves Acuáticas de enero de 1972, se señalan sendas concentraciones de aprox. 17.000 aves, respectivamente, el 21.I en Fuentedepiedra (Málaga), (Tamisier en BERNIS 1972) y el 19.II en Gallocanta (Zaragoza), (Araújo \& Landín en la misma obra), que posiblemente se tratase del mismo. Nosotros, aparte de los resultados que se pueden ver en los censos, 10 vemos raro un determinado día, y a la semana siguiente, por el contrario, notablemente abundante. Por lo tanto, un bando de $A y$. ferina que hoy se ve en «El Hondo» puede estar, dentro de unos cuantos días, en la Albufera de Valencia o las Tablas de Daimiel, lo que explicaría, en nuestra opinión, las fuertes fluctuaciones que sufre aquí de una fecha a otra.

MARTORELL vio algunas hembras con pulls. y un nido con cinco huevos, en junio 1965. Nosotros vemos todos los veranos hembras seguidas de polluelos -4 a 9 , normalmente-, nadando o cruzando el camino. A mediados y finales de marzo se ven los corros de cortejo (NAVARRO 1972): una hembra es asediada por dos, cuatro o seis machos que se atacan ritualmente entre sí por algún tiempo, hasta que alguno de ellos consigue ser seguido por la hembra en cuestión.

Máximas cantidades - tanto para época de nidificación como para la invernal- en: 7.03.71, 80 spms.; $11.01 .73,3.560$ spms. en todo el coto; 17.06.73, 60 spms. en la Charca Sur (¿mancones?); 28.10.73, 8.200 spms. en todo el coto, la mayoría en el embalse de Levante. Posiblemente migrantes sedimentados; $25.11 .73,3.850$ spms. en todo; 17.02.74, unos 2.500 spms. sólamente en la Charca Sureste; 25.01.75, 1.100 spms.; 18.01.76, 6.100 spms.; 21.03.76, 556 spms.; y 6.11.77, unos 3.000 spms. tan sólo en la Charca Sureste.

Datos sobre un ave anillada, cazada en el coto: ANILLAMIENTO: 15-03-68, Lucerna (Suiza). 


\section{CAPTURA: $\quad 00.12 .73$, «El Hondo» (Alicante). \\ No. ANILLA Y CENTRO: 26688 - Sempach.}

En general, especie común que se ve medianamente durante todo el año y en gran abundancia en invierno. Durante los días de cacería, al igual que Netta, algunos huyen a las cercanas Salinas de la Mata, aunque no en tanta cantidad como aquél.

: Aythya nyroca, Porrón Pardo.-(Roget)

Especie escasísima, posiblemente nidificante y sedentaria, pero que por su rareza y costumbres sólo la hemos podido observar en contadas ocasiones.

En mayo de 1971, vimos en dos diferentes fechas a una hembra muy aquerenciada al mismo punto, en el embalse de Poniente, lo que podría haber significado un nido cercano (NAVARRO 1972).

Además, dos observaciones invernales. El 19.11.72, una hembra recién cazada (embalse de Poniente), y el 11.01.73, dos machos muy bien vistos por largo rato, cerca del camino, en el mismo embalse (Navarro y otros en ARAÚJO \& $G^{a}$ RUA 1973).

Desde entonces no lo hemos vuelto a ver.

Aythya fuligula, Porrón Moñudo.-(Matruca)

De presencia escasa como invernante, más una cita en junio:

26.11.72. - una hembra recién cazada, embalse de Poniente.

03.06.73. - un macho nadando en el embalse de Levante y otro en el Poniente, entre $A y$. ferina.

28.10.73. - un macho en el embalse de Levante, entre $A y$. ferina.

25.01.75. - 4 smps. en la Charca Sur y 3 en Levante.

18.01.76.-3 spms. en la Charca Sur.

Sin duda, los embalses de «El Hondo» no se hallan dentro de su área de invernada en España. 
La única cita, la recogida por TRIGO DE YARTO (1960), señalando que fue cazada aquí en diciembre de 1959. Hemos preguntado a los guardas del coto, mostrándoles dibujos y fotos de esta especie, y aseguran no conocerla.

Pandion haliaetus, Águila Pescadora.-(Idem)

mP. mO.

Únicamente durante ambos pasos, presentándose regularmente en los meses de marzo y septiembre de todos los años. Normalmente se ve a un sólo individuo, pero algunas veces han coincidido dos el mismo dia. Se las ve volando bajo sobre el agua a la captura de peces; se posan a descansar en los postes telefónicos o en las estaquillas que existen en medio del agua. El 27.03.74, pudimos observar a un spm. que se hallaba posado sobre el tejado de una caseta, en la orilla de la Charca Norte. Sale volando con una gran carpa entre las garras, perseguido incansablemente por una Larus argentatus que lo acosó durante largo rato.

Circus aeruginosus, Aguilucho Lagunero.-(Águila Ratera)

Especie que, hasta hace unos tres años, se veía durante todo el año en los embalses, donde posiblemente nidificaba en cantidad reducida (3-4 parejas) (NAVARRO 1972).

Desde el comienzo de nuestro estudio ha disminuido considerablemente su población, pues en la actualidad sólo se la ve durante el invierno y en escaso número. Éso puede ser debido, como desgraciadamente ocurre en otras especies de rapaces en todos los países, a los efectos nocivos de los biocidas, a los cuales las aves de presa son muy sensibles por ser los últimos eslabones de la cadena trófica y que, si bien no les causan la muerte en todos los casos, sí consiguen la infecundidad de los huevos.

Siempre se le ve volando bajo sobre el carrizal o sobre el agua, contra el viento, al acecho de pequeños pajarillos y otros vertebrados. En NAVARRO 1972 ya dimos algunos datos sobre posible alimentación a base de Gallinula chloropus.

En sus buenos tiempos de abundancia, se podían ver hasta 8 spms. 
en vuelo conjunto de remonte (p. ej.: 7.11.71). También era abundante en las cercanas Salinas de Santa Pola, donde también vimos en una ocasión 8 hembras volando juntas.

: Rallus acuaticus, Rascón.-(Picoruda)

Común y sin duda nidificante, aunque por ser una especie de hábitos sumamente escondedizos y retraídos y construir el nido en lo más intrincado del carrizal, no hemos podido descubrir ninguno.

Ya en pleno invierno se observan parejas que se persiguen emitiendo gruñidos, por lo que la nidificación debe comenzar a finales de febrero.

Es la especie más cazada puesto que, al contrario que las anátidas -principalmente Netta. y Ay. ferina-, que con los primeros disparos huyen a refugiarse a las diversas salinas, cercanas, Fulica permanece en este lugar, aunque la gran mayoría se refugian en el carrizal.

La mayoría de los años ya se ven nidos con puestas a primeros de marzo -excepto el de 1974, en que se retrasó un mes el comienzo de la nidificación de casi todas las especies-. Adultos con pulls. a mediados del mismo mes.

Construye nidos mayores que Gallinula, más descuidadamente hechos, y principalmente a base de tallos de carrizos - Gallinula lo hace con las hojas-, normalmente en el espeso carrizal y suspendidos sobre el agua, aunque vemos en raras ocasiones nidos flotantes, entre las colonias de Chlidonias.

Puesta media de 7-8 huevos, más blancos que los de Gallinula, con pintas más pequeñas y negras. Durante los meses de abril, mayo, junio y julio, abundan las observaciones de adultos seguidos de polluelos.

Alimentación, según observaciones propias, básicamente vegetal, aunque con mayor porcentaje de insectos en primavera y verano. Totalmente fitófaga durante el invierno.

En el invierno es muy abundante. Así, ya lo citaron MARTORELL («muy abundante en invierno y verano»), TATO («muy abundante») y P. Pascual en BERNIS 1964 (invierno 1953-54: «quizá unas cinco mil»). 
Las fechas de mayor abundancia, según datos propios, son las siguientes: 8.12.69: miles de individuos, en el embalse de Poniente (NAVARRO 1972); 11.01.73, 3.800 en total; 27.07.73, unas 800, sólo en la Charca Norte; 9.09.73, cerca de 1.000 en el mismo sitio; 13.09.73, más de 12.000 en todo el coto - migrantes sedimentados, sin duda-; 25.11.73, casi 8.500 en todo el coto; 17.02 .74 , abundantísima por doquier; $25.01 .75,4.700$ en total; $18.01 .76,6.000$ en total; 21.03.76, 1.000 en total - ya habían partido los invernantes-.

En nuestra opinión, la mayoría de las Fulica invernantes en «El Hondo» deben deben provenir de otras zonas españolas. Todas las recuperadas anilladas en el invierno de 1973, fueron marcadas como pull. en las Marismas del Guadalquivir. De vez en cuando se recupera alguna anillada en el extranjero. MARTORELL cita una de ellas, anillada de Letonia en mayo-63 y recuperada aquí en enero-64.

Grus grus, Grulla Común.-

Únicamente la cita ya referida en Ardeola (NAVARRO 1975), de un spms. en la Charca Sur.

Charadrius dubius, Chorlitejo Chico.-

$\mathrm{mO}$

Como única cita, tenemos la de bastantes migrantes sedimentados, el 26.09.71 (NAVARRO 1972), posándose en las zonas secas del embalse de Poniente, que en aquella fecha tenía muy poca agua.

X Charadrius alexandrinus, Chorlitejo Patinegro.-

$\mathrm{E}$

Observaciones únicamente estivales, referidas a la zona de saladares de la parte NW de la Charca Norte.

Alli deben anidar unas 8-10 parejas de esta especie, según recuentos efectuados de 1973 a 1977. El 19.5.74, vemos un nido con 3 huevos, que son incubados por la hembra.

También anida en gran cantidad en las Salinas de Santa Pola y de La Mata.

Como dato curioso, citamos la observación de 4 spms. posados sobre la ova flotante, en la Charca Sur-Este, el día 17.06.73. 


\section{AGRADECIMIENTOS}

Queremos agradecer la colaboración y facilidades que nos ha prestado todo el personal de la Compañía de Riegos de Levante, S. A., en la actualidad Comunidad de Riegos de Levante-Márgen izquierda, y principalmente a los Directores de ambas, Don Rafael Miró Silvestre y Don José María Berenguer Mateu, respectivamente, por los medios puestos a nuestra disposición.

A Don Ginés Torres, Don Manuel Berenguer, Don Salvador Maciá y Don José Torres, trabajadores de «El Hondo», nuestro profundo agradecimiento y nuestra eterna amistad, por las horas empleadas en llevarnos, a golpe de pértiga, por los más recónditos y difíciles carrizales de los embalses y, además, por habernos transmitido sus grandes conocimientos sobre la avifauna del lugar.

Murcia, Nov. 77 


\section{BIBLIOGRAFÍA}

ARAÚJO PONCIANO, J. \& GARCÍA RÚA, A.E. (1973): El censo español de aves acuáticas de enero de 1973. Boletín Estación Central de Ecología, año II, n 4: 11-39.

BERNIS MADR.AZO, F. (1964): Información española sobre anátidas y Fochas (época invernal). Sociedad Española Ornitología.

MARTORELL, M. (1966): Nota sobre la avifauna de la laguna del Hondo, en Elche. Ardeola 11: 129-136.

CÍA DE RIEGOS DE LEVANTE, S. A.: (1973): Plan de conservación $y$ aprovechamiento cinegético del coto privado de «El Hondo». Elche (circulación restringida).

CORONADO, R., DEL PORTILlO, F. \& SÁEZ-ROYUELA, R. (1973): Guía de las anátidas de España. Publicaciones ICONA, Madrid.

MARTORELL, M. (1966): Nota sobre la avifauna de la laguna del Hondo, en elche. Ardeola 11: 129-136.

NAVARRO MEDINA, J. D. (1971 b): Notas preliminares sobre aves acuáticas de las Salinas de Santa Pola (Alicante). Ardeola 15: 91-93.

(1971 c): Observación de Anas angustirostris en la provincia de Alicante, Ardeola 15: 126.

- - - (1972): Panorama ornitológico de los embalses de «El Hondo» (Alicante). Ardeola 16: 228-239.

PALAUS SOLER, F. J. (1959): Notas ornitológicas del Nor-Este de España (con algunos datos de Levante). Ardeola 6 (1): 222.

TATO CUMMING, J. J.: (1960): Notas invernales de la zona costera del Sur-Este de España, Ardeola 6 (2): 362-363.

TRIGO DE YARTO, E. (1960): Notas sobre capturas de aves raras o interesantes. Ardeola 6 (2): 367-369. 